\title{
BMJ open Changes in somatic disease incidents during opioid maintenance treatment: results from a Norwegian cohort study
}

\author{
Ivar Skeie, ${ }^{1,2}$ Mette Brekke, ${ }^{3}$ Michael Gossop, ${ }^{2,4}$ Morten Lindbaek, ${ }^{3,5}$ \\ Even Reinertsen, ${ }^{6}$ Magne Thoresen, ${ }^{7}$ Helge Waal ${ }^{2}$
}

To cite: Skeie I, Brekke M, Gossop M, et al. Changes in somatic disease incidents during opioid maintenance treatment: results from a Norwegian cohort study. BMJ Open 2011;1:e000130. doi:10.1136/bmjopen-2011000130

- Prepublication history for this paper is available online. To view these files please visit the journal online (http:// bmjopen.bmj.com).

Received 28 March 2011 Accepted 7 June 2011

This final article is available for use under the terms of the Creative Commons Attribution Non-Commercial 2.0 Licence; see http://bmjopen.bmj.com

For numbered affiliations see end of article.

Correspondence to

Dr Ivar Skeie;

ivskeie@online.no

\section{ABSTRACT}

Objectives: To examine the effect of opioid maintenance treatment (OMT) on somatic morbidity in a cohort of OMT patients.

Design: Retrospective cohort study.

Setting: OMT programme in two Norwegian counties. Participants: 200 OMT patients, participation rate $71.2 \%$.

Main outcome measures: Incidence rates (IR) before, during and after OMT for acute/subacute hospital-treated somatic disease incidents (drugrelated, non-drug-related, injuries) and rates for inpatient days and outpatient treatment contacts.

Results: IR for drug-related hospital treatment episodes were $76 \%$ lower during compared to before OMT (before versus during incidence rate ratio (IRR) $4.2(95 \% \mathrm{Cl} 2.9$ to 6.2$), \mathrm{p}<0.001)$ and 11 times higher after compared to during OMT (after versus during IRR 11.1 (6.6 to 18.5), $p<0.001$ ). For non-drug-related treatment episodes, IR were $35 \%$ higher during than before OMT (before versus during IRR 0.7 (0.6 to 1.0), $\mathrm{p}=0.02$ ) and $32 \%$ higher after compared to during OMT (IRR 1.4 (0.9 to 2.2), $p=0.15$ ), while injuries showed little change according to OMT status. Although patients with on-going drug-taking during OMT showed less reduction in drug-related hospital-treated incidents during treatment than patients not using illicit drugs, the quartile with most drug-taking showed a significant reduction (before versus during IRR 3.6 (2.4 to 5.3)). Patients who had experienced cessation of OMT showed a significant reduction in drug-related treatment episodes during OMT (before versus during IRR 1.7 (1.0 to 2.9)), although less than patients without OMT interruptions (before versus during IRR 6.1 (3.6 to 10.6)), and a significant increase after OMT cessation compared with during OMT (IRR 5.4 (3.0 to 9.7)).

Conclusion: Acute/subacute drug-related somatic morbidity is reduced during compared to before OMT. This was also found for patients with on-going drugtaking during OMT. However, acute drug-related health problems show an increase after OMT cessation, and this is a matter of concern. Further studies on somatic morbidity after OMT cessation should be carried out.

\section{ARTICLE SUMMARY}

Article focus

- Opioid maintenance treatment (OMT) is the most widely used treatment for opioid dependence, but the effects of OMT on physical health problems have received relatively little attention.

- This study investigates how acute somatic morbidity (drug-related, non-drug-related, injuries) varies according to OMT status (before, during, after OMT) in a cohort of 200 OMT patients.

- The research questions were: Is somatic morbidity reduced during OMT compared to before and after treatment? If so, what types of disease incidents are reduced? How is the effect of OMT status on somatic morbidity influenced by various patient characteristics?

\section{Key messages}

- Acute drug-related somatic morbidity (overdoses, injecting-related, other) is substantially reduced during compared to before OMT.

- This was also found for 'problem patients' with on-going drug-taking during OMT, but to a lesser degree than for patients not using illicit drugs.

Strengths and limitations of this study

Strengths

- Participation rate was high, selection bias limited observation period long and the evaluation of morbidity changes was based on assessment of full-text hospital records.

\section{Limitations}

- The study focused on acute health problems treated in hospital, but elective hospital contact due to chronic health problems and primary healthcare contacts were not included.

\section{INTRODUCTION}

Dependent opioid users, especially those injecting heroin, have increased somatic ${ }^{1-3}$ and psychological morbidity ${ }^{3-6}$ and reduced health-related quality of life. ${ }^{78}$ Injecting drug users are prone to chronic bloodborne viral infections, especially HIV/AIDS ${ }^{9} \quad{ }^{10}$ and chronic hepatitis $\mathrm{B}^{11}$ and hepatitis $\mathrm{C},{ }^{11}{ }^{12}$ as 
well as acute and subacute bacterial infections and other complications related to injecting. ${ }^{13}$ Opioid dependence is associated with social marginalisation, criminality and socioeconomic deprivation accompanied by malnutrition, chronic diseases and generally impaired health as well as exposure to overdoses and trauma. ${ }^{1}$ Mortality among injecting drug users is much greater than in the general population, with a standardised mortality ratio ranging from 5 to 30 in studies from several countries. ${ }^{10}{ }^{14}$ The main causes of death (in descending order) are overdose, diseases, trauma and suicide. ${ }^{10}$

Maintenance treatment, hereafter called opioid maintenance treatment (OMT), has been the most widely used treatment for opioid dependence for the last number of decades. ${ }^{15}$ The specific changes in physical and mental health that occur during OMT have received surprisingly little detailed research attention. ${ }^{16}$ Maintenance treatment leads to reduced use of illegal opioids and less injection. ${ }^{17}{ }^{18}$ It also induces tolerance to opioids, ${ }^{19}{ }^{20}$ and a corresponding decline in drugrelated morbidity including overdoses should be expected. Some studies report improved somatic health during OMT based on interviews, ${ }^{17}$ clinical assessment ${ }^{21}$ and reduced consumption of inpatient care due to infections, ${ }^{22}{ }^{23}$ but relatively few studies have investigated OMT-related somatic health effects and morbidity patterns. In a previous study we found reductions in somatic disease incidents treated in hospital or by general practitioners during OMT compared to "not in treatment'. ${ }^{24}$ Drug-related incidents were reduced by about two thirds, but non-drug-related incidents showed a non-significant increase (possibly due to closer contact with health services) and injuries showed no change during treatment. These findings were, however, based upon a small sample of patients from one municipality. In order to evaluate the effects of maintenance treatment, it is necessary to study morbidity prior to, during and after OMT. Such studies are scarce, and very few include long-term follow-up.

The present study investigates how somatic co-morbidity varies according to OMT status (before, during and after OMT) in a group of 200 patients. The main hypothesis to be tested was that somatic morbidity is reduced during OMT. More detailed research questions were: (1) What changes in somatic morbidity are found during OMT compared to before and after treatment, and what types of disease incidents are reduced? and (2) How is the effect of OMT status on somatic morbidity influenced by various patient characteristics?

\section{MATERIALS AND METHODS}

Design, sample and setting

Methadone and buprenorphine are used as substitution medications in the national Norwegian OMT programme, which began in 1998. On 13 December 2009,5383 people were in treatment, and $55.7 \%$ were receiving methadone and $44.3 \%$ buprenorphine. ${ }^{25}$
Norway has a population of 4.9 million and the target group for OMT is estimated to be about $10000 .^{25}$

The study has a retrospective cohort design. The cohort was established in 2007-8 and consists of those admitted to OMT from 1998 until the end of June 2007 in two counties (Hedmark and Oppland) in Norway (figure 1). The participants were recruited through their treatment contacts. Out of a total of 319 patients who started OMT, 38 had no contact with local health or social services at the time of invitation and were regarded as ineligible for this study. Of the remaining 281, 13 who died after their first OMT entry were included. Among the 268 eligible subjects still alive, 187 consented to participate and 81 did not, and so the cohort consisted of 200 persons. The participation rate was $71.2 \%, 68.8 \%$ among patients in treatment versus $73.7 \%$ among those not in treatment when invited.

\section{Measures}

A somatic disease incident was defined as any acute or subacute health problem leading to inpatient or outpatient hospital treatment, henceforth called hospital treatment episodes, treatment episodes or just episodes. The numbers of hospital treatment episodes, inpatient days and outpatient hospital contacts were recorded. Incidence rates and rates of inpatient days and outpatient contacts for the periods before, during and after OMT were calculated. Incidence rate ratios before versus during treatment and after versus during treatment were estimated. Only records from somatic departments were examined. Psychiatric disease incidents were only considered if they caused a somatic condition, for example due to self-harm. Hospital contacts for chronic somatic disorders were not included, but acute treatment episodes caused by an underlying chronic disease were assessed. One episode could lead to more than one contact, for instance follow-up of a fracture or an abscess. One episode documented in records from several hospitals was only counted once. Episodes were

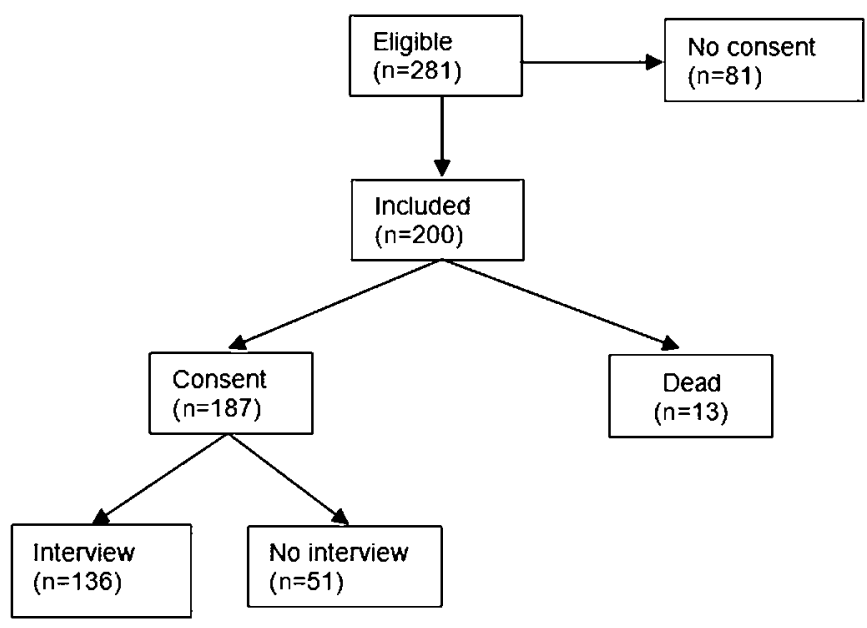

Figure 1 Participants: patients starting opioid maintenance treatment between 1998 and June 2007. The studied cohort consists of the 200 included patients. 
categorised as drug-related (overdoses, injecting-related, other), non-drug-related (infection, other) or injuries.

\section{Data}

One hundred and thirty-six of the 187 alive participants $(73 \%)$ underwent structured interviews which collected information on personal data, former hospital contacts and drug history as well as education and employment history.

Records from somatic departments in local hospitals were examined for all participants. Based on interview information, records from other hospitals were collected and more than $99 \%$ of all requested records were examined. Only incidents of somatic disease documented in hospital records were included and records for all 200 participants were examined.

Data were also drawn from annual status reports on each patient in the national OMT programme with information about ongoing drug-taking during OMT based on urine tests and clinical assessment. A combined score based on use of illicit opioids, cannabis, benzodiazepines and central stimulants during OMT was calculated for each patient. Data were obtained for 183 participants $(91.5 \%)$.

\section{Observation period}

Data were studied for the 5 years before first admittance to maintenance treatment, up to the first 5 years during OMT (one or consecutive periods), and up to the first 5 years out of treatment (one or consecutive periods) after first admittance to OMT. Thus, the post-OMT period was defined as the sum of the time between treatment periods and time after the last treatment period. The total observation period was 1000 patientyears before, 813 during and 91 after OMT. The date of collection of their record from the local hospital (during 2008-9) was defined as the study end-point for each patient.

\section{Inter-rater agreement}

Inter-rater agreement was established in the pilot study. ${ }^{24}$ We found high inter-rater reliability for whether hospital treatment episodes were drug-related $(\kappa=1)$ and for categories among drug-related treatment episodes (overdose, injecting-related or other, $\kappa=0.82$ ). In this study, IS (the first author) first scrutinised all full-text records. Treatment episodes considered difficult to categorise were discussed between IS and another physician (the co-author ER) until consensus was reached.

\section{Statistical analyses}

Incidence rates were analysed by means of a Poisson regression model. Dependencies in the data, due to the fact that each participant was measured repeatedly (before, during and after OMT), were handled by generalised estimating equations with unstructured working correlation and robust variance estimation. With regard to drug-related treatment episodes, we investigated the possible influence of different patient characteristics on the effect of OMT by including the interaction between OMT and the characteristic in question in the model, one by one. Incidence rate ratios with $95 \%$ CIs were estimated. The significance level was set to 5\%. All analyses were performed in SPSS v 15 .

The study was approved by the Regional Committee for Medical Research Ethics and the Norwegian Data Inspectorate. Exemption from the duty of confidentiality and professional secrecy for those who had died was granted by the Norwegian Directorate of Health.

\section{RESULTS}

\section{Cohort characteristics}

Sixty-six per cent of the cohort were men and the mean age at the first entry to maintenance treatment was 37 years. Methadone was prescribed to $67 \%$ and buprenorphine to $33 \%$ at the point of entry to the study. Mean dosage for methadone was $122 \mathrm{mg}$ and $17.6 \mathrm{mg}$ for buprenorphine. Fifty-one out of 200 (26\%) had left maintenance treatment once or more during the observation period, while the rest had been in continuous treatment since they were included. Mean age of first use of heroin was 21.1 years, and mean duration of opioid dependence before OMT was 12.3 years. Thirtyfour per cent reported more than 10 overdoses during their lifetime, $54 \%$ between one and 10 , and $12 \%$ reported none. Seventeen per cent had not completed 9 years of compulsory schooling, $50 \%$ had completed 9 years and $33 \%$ had 12 or more years of education. Twenty-three per cent had $<1$ year of employment, $28 \%$ between 1 and 5 years and $49 \%$ had more than 5 years of work experience.

Among the patients who had experienced interruption of maintenance treatment, $15 \%$ were assessed as stable and drug-free when leaving OMT for the first time, while $85 \%$ were assessed as unstable and taking drugs.

\section{Changes in hospital-treated somatic disease incidents}

Table 1 shows the rates of hospital treatment episodes due to acute and subacute somatic disease incidents and the rates for inpatient days and outpatient hospital contacts for the various categories of episodes before, during and after OMT. Table 2 displays the statistical significance of these changes by showing the incidence rate ratios according to different OMT status; before versus during OMT and after versus during OMT, respectively. A total of 1021 somatic disease incidents were registered: 605 before, 310 during and 106 after OMT.

The rate of all treatment episodes was $37 \%$ lower during compared to the period before treatment (before versus during OMT incidence rate ratio (IRR) 1.6 (95\% CI 1.3 to 1.9$), \mathrm{p}<0.001)$. The rate in the post-OMT period was $197 \%$ higher compared to the period during OMT (after versus during treatment IRR 2.8 (95\% CI 2.1 to 3.9$), \mathrm{p}<0.001)$.

During treatment, the rate of all inpatient hospital treatment days was 38\% lower (before versus during 
Table 1 Acute/subacute hospital-treated somatic disease incidents: incidence rates and rates of inpatient days and outpatient treatment contacts per 100 patient-years

\begin{tabular}{|c|c|c|c|}
\hline & \multicolumn{3}{|c|}{ OMT status } \\
\hline & Before & During & After* \\
\hline \multicolumn{4}{|l|}{ Incidence rates } \\
\hline All acute/subacute incidents & 60.5 & 38.1 & 113.0 \\
\hline All drug-related incidents & 31.9 & 7.5 & 75.7 \\
\hline Overdoses & 10.4 & 3.7 & 20.8 \\
\hline Injecting-related, total ${ }^{\star \star}$ & 14.2 & 2.4 & 34.0 \\
\hline Deep venous thrombosis/lung embolism & 0.6 & 0.2 & 1.1 \\
\hline Acute hepatitis $B$ and $C$ & 0.9 & 0.1 & 1.1 \\
\hline Local bacterial infections & 8.5 & 1.5 & 19.7 \\
\hline Systemic bacterial infections & 3.3 & 0.5 & 11.0 \\
\hline Other & 0.9 & 0.1 & 1.1 \\
\hline Other drug-related, total ${ }^{\star *}$ & 7.3 & 1.4 & 20.8 \\
\hline Withdrawal-related & 2.7 & 0.5 & 4.4 \\
\hline Impaired general condition & 1.4 & 0.2 & 7.7 \\
\hline Neuromuscular & 0.5 & 0.0 & 1.1 \\
\hline Other & 2.7 & 0.6 & 7.7 \\
\hline All non-drug-related incidents & 12.4 & 16.7 & 21.9 \\
\hline Infections & 3.7 & 4.7 & 11.0 \\
\hline Other & 8.7 & 12.1 & 11.0 \\
\hline Injuries & 16.2 & 13.9 & 15.4 \\
\hline \multicolumn{4}{|l|}{ Treatment contact rates } \\
\hline Inpatient days per 100 patient-years & 150.7 & 92.4 & 472.8 \\
\hline Drug-related & 86.9 & 13.9 & 331.3 \\
\hline Non-drug-related & 31.7 & 62.9 & 69.1 \\
\hline Injuries & 32.1 & 15.6 & 72.4 \\
\hline Outpatient contacts per 100 patient-years & 56.4 & 41.4 & 107.5 \\
\hline Drug-related & 22.9 & 4.8 & 49.4 \\
\hline Non-drug-related & 12.1 & 16.9 & 26.3 \\
\hline Injuries & 21.4 & 19.8 & 31.8 \\
\hline \multicolumn{4}{|c|}{$\begin{array}{l}\text { The rates before and during OMT refer to all patients, while the rates after OMT refer exclusively to patients with interrupted OMT. Patient-years } \\
\text { at risk: } 1000 \text { before, } 813 \text { during and } 91 \text { after OMT. Number of incidents: } 605 \text { before, } 310 \text { during and } 106 \text { after OMT. } \\
{ }^{\star} \text { After OMT is defined as time out of treatment (one or more consecutive periods) after first admittance to OMT, that is the sum of the time } \\
\text { between treatment periods and time after the last treatment period. } \\
\star^{\star} \text { Subgroups in italic. } \\
\text { OMT, opioid maintenance treatment. }\end{array}$} \\
\hline
\end{tabular}

treatment IRR $1.7(95 \%$ CI 1.1 to 2.4$), \mathrm{p}=0.01)$ and the overall rate of outpatient hospital treatment contacts was $27 \%$ lower (before versus during treatment IRR 1.4 (95\% CI 1.0 to 1.8$), \mathrm{p}=0.04)$. After treatment the rate of inpatient days was 5.1 times higher than during treatment (IRR 5.9 (95\% CI 3.6 to 9.6), p<0.001) and the rate of outpatient hospital treatment was 2.6 times higher (IRR 2.0 (95\% CI 1.1 to 3.8$), \mathrm{p}=0.03)$.

\section{Drug-related disease incidents}

The rate of drug-related hospital treatment episodes was $76 \%$ lower during treatment than before (before versus during IRR 4.2 (95\% CI 2.9 to 6.2), $\mathrm{p}<0.001)$. The rate of injecting-related episodes was $83 \%$ lower (IRR $5.9(95 \%$ CI 3.1 to 11.4$), \mathrm{p}<0.001)$ and the rate of overdoses was $64 \%$ lower (IRR 2.8 (95\% CI 1.6 to 5.0$)$, $\mathrm{p}<0.001)$ than before OMT. Among the injecting-related episodes, local and systemic bacterial infections were the most common. The rate of drug-related inpatient days was $84 \%$ lower (IRR 6.3 (95\% CI 3.4 to 11.8$), \mathrm{p}<0.001$ ) and the rate of outpatient contacts was $79 \%$ lower during treatment compared to the pre-treatment period (IRR $4.8(95 \%$ CI 2.7 to 8.3$), \mathrm{p}<0.001)$.

The post-OMT rate for drug-related treatment episodes was higher than the rate before or during treatment. Compared to the period during OMT, the rate was 10.1 times higher (IRR 11.1 (95\% CI 6.6 to 18.5), $\mathrm{p}<0.001)$. Overdoses after OMT were double those before OMT and the overdose rate was 5.6 times higher compared to the period during OMT (IRR 5.8 (95\% CI 2.7 to 12.3$), \mathrm{p}<0.001)$. The rate of injectingrelated episodes was 14.2 times higher after than during treatment (IRR 12.6 (95\% CI 4.3 to 36.8), p<0.001), the rate of inpatient days due to drug-related episodes was 23.8 times higher (IRR 25.2 (95\% CI 13.1 to 48.7), $\mathrm{p}<0.001)$ and the rate of outpatient contacts was 10.3 times higher (IRR 10.6 (95\% CI 4.7 to 25.9 ), $\mathrm{p}<0.001)$.

\section{Non-drug-related disease incidents}

Non-drug-related treatment episodes were $35 \%$ more frequent during treatment compared to the pre-OMT 
Table 2 Acute/subacute hospital-treated somatic disease incidents: crude incidence rate ratios and treatment contact rate ratios of inpatient days and outpatient hospital contacts

\section{IRR/TCRR (95\% CI) p Value}

\begin{tabular}{lll}
\hline $\begin{array}{l}\text { Before versus during OMT, during OMT as reference } \\
\text { (incidence rate=1) }\end{array}$ & \\
All incidents & $1.6(1.3$ to 1.9$)$ & $<0.001$ \\
Drug-related* & $4.2(2.9$ to 6.2$)$ & $<0.001$ \\
Overdoses & $2.8(1.6$ to 5.0$)$ & $<0.001$ \\
$\quad$ Injecting-related & $5.9(3.1$ to 11.4$)$ & $<0.001$ \\
$\quad$ Other & $5.7(2.8$ to 11.9$)$ & $<0.001$ \\
Non-drug-related & $0.7(0.6$ to 1.0$)$ & 0.02 \\
Injuries & $1.2(0.9$ to 1.5$)$ & 0.23 \\
Inpatient days & & \\
Drug-related & $6.3(3.4$ to 11.8$)$ & $<0.001$ \\
Non-drug-related & $0.5(0.3$ to 1.0$)$ & 0.04 \\
Injuries & $2.1(0.7$ to 5.7$)$ & 0.17 \\
All & $1.7(1.1$ to 2.4$)$ & 0.01 \\
Outpatient contacts & & \\
Drug-related & $4.8(2.7$ to 8.3$)$ & $<0.001$ \\
Non-drug-related & $0.7(0.4$ to 1.2$)$ & 0.18 \\
Injuries & $1.1(0.7$ to 1.6$)$ & 0.78 \\
All & $1.4(1.0$ to 1.8$)$ & 0.04
\end{tabular}

After versus during OMT, during OMT as reference (incidence rate $=1$ )

\begin{tabular}{|c|c|c|}
\hline Alincidanto & & \\
\hline All incidents & $2.8(2.1$ to 3.9$)$ & $<0.001$ \\
\hline Drug-related & 11.1 (6.6 to 18.5$)$ & $<0.001$ \\
\hline Overdoses & 5.8 (2.7 to 12.3$)$ & $<0.001$ \\
\hline Injecting-related & 12.6 (4.3 to 36.8$)$ & $<0.001$ \\
\hline Other & 16.7 (6.5 to 42.7$)$ & $<0.001$ \\
\hline Non-drug-related & $1.4(0.9$ to 2.2$)$ & 0.15 \\
\hline Injuries & 0.8 (0.3 to 1.8$)$ & 0.57 \\
\hline \multicolumn{3}{|l|}{ Inpatient days } \\
\hline Drug-related & $25.2(13.1$ to 48.7$)$ & $<0.001$ \\
\hline Non-drug-related & 1.1 (0.5 to 2.3$)$ & 0.78 \\
\hline Injuries & $4.6(0.8$ to 28.5$)$ & 0.98 \\
\hline All & 5.9 (3.6 to 9.6$)$ & $<0.001$ \\
\hline \multicolumn{3}{|l|}{ Outpatient contacts } \\
\hline Drug-related & 10.6 (4.7 to 25.9 ) & $<0.001$ \\
\hline Non-drug-related & $1.6(0.7$ to 3.7$)$ & 0.26 \\
\hline Injuries & $1.1(0.4$ to 3.1$)$ & 0.87 \\
\hline All & $2.0(1.1$ to 3.8$)$ & 0.03 \\
\hline
\end{tabular}

The incidence rate ratios indicate the statistical significance of the changes in incidence rates demonstrated in table 1, estimated by Poisson regression (generalised estimating equations). Patientyears at risk: 1000 before, 813 during and 91 after OMT. Number of incidents: 605 before, 310 during and 106 after OMT.

IRR, incidence rate ratios; OMT, opioid maintenance treatment; TCRR, treatment contact rate ratios.

*Subgroups in italic.

period (before versus during IRR 0.7 (95\% CI 0.6 to 1.0 ), $\mathrm{p}=0.02$ ) and both infections and other non-drug-related episodes increased. After treatment there was a $31 \%$ increase for all non-drug-related episodes versus the period during OMT (IRR 1.4 (95\% CI 0.9 to 2.2), $\mathrm{p}=0.15$ ). The rates for inpatient days and outpatient hospital treatment contacts showed similar changes.

\section{Injuries}

The changes in incidence and treatment contact rates for injuries were minor and non-significant.
Interaction between OMT status and patient characteristics From the data in table 1 it is evident that changes according to OMT status in the total number of hospital treatment episodes are associated with changes in drugrelated treatment episodes. In the analysis of how the effect of OMT status is influenced by various patient characteristics, we therefore concentrated on drugrelated episodes alone. Table 3 shows the interaction between the effect of maintenance treatment (OMT status) and various patient characteristics, that is the incidence rate ratio between the periods before and during OMT for drug-related treatment episodes adjusted for these characteristics.

In a comparison of patients who received continuous maintenance treatment throughout the during-OMT observation period versus those who left treatment once or more, those with continuous treatment showed greater incidence rate reduction during treatment than those with interrupted treatment, although the latter still showed significant reduction versus the pre-treatment period. After treatment, the group with interrupted treatment showed an increase, with the incidence rate ratio between the periods after and during OMT for this group being 5.4 (3.0 to 9.7) (not shown in the table). Patients with ongoing illicit drug-taking during OMT showed less reduction in incidence rate during treatment than patients not using illicit drugs. Even so, the quartile taking most drugs showed a significant reduction during versus before OMT (before versus during IRR ratio 3.6 (2.4 to 5.3)).

Among the pre-OMT patient characteristics, only employment history and years of opioid dependence before OMT showed a significant interaction with OMT status in the period before versus during OMT. Individuals with less work experience and fewer years of opioid dependence, respectively, showed less reduction in incidence rates during compared to before treatment. Interaction was also tested for gender, age at OMT start, lifetime number of overdoses, years of education and age at heroin debut without showing any statistically significant interaction $(\mathrm{p}>0.1)$.

\section{DISCUSSION}

The study shows a substantial reduction in drug-related hospital-treated incidents of somatic disease during maintenance treatment compared to the pre-treatment period. The reduction consists of less overdoses, and fewer injecting-related and other drug-related treatment episodes.

Overdoses are the most frequent cause of death among dependent opioid users, ${ }^{10}$ and the $64 \%$ reduction in overdoses during treatment is an important finding. Several studies have documented reduced mortality during OMT compared to the pre-OMT period ${ }^{9} 26-32$ and after leaving OMT, ${ }^{33-35}$ and the reduction in overdoses found in the present study supports previous findings of reduced mortality during OMT. 
Table 3 Acute/subacute drug-related hospital-treated somatic disease incidents: interaction effect between OMT status (before versus during OMT) and patient characteristics

\begin{tabular}{|c|c|c|}
\hline Patient characteristics & p Value for interaction & Adjusted IRR $(95 \% \mathrm{Cl})$ \\
\hline \multicolumn{3}{|l|}{ During-treatment characteristics } \\
\hline Interruption of OMT & 0.001 & \\
\hline Continuous treatment & & 6.1 (3.6 to 10.6$)$ \\
\hline Interrupted treatment & & 1.7 (1.0 to 2.9$)$ \\
\hline $\begin{array}{l}\text { Drug-use during OMT (illicit opioids, cannabis, } \\
\text { benzodiazepines, central stimulants) }\end{array}$ & 0.07 & \\
\hline 25th Percentile, quartile without drug use & & 6.5 (3.5 to 12.0$)$ \\
\hline 75th Percentile, quartile with most drug use & & $3.6(2.4$ to 5.3$)$ \\
\hline \multicolumn{3}{|l|}{ Pre-treatment characteristics } \\
\hline Employment years & 0.02 & \\
\hline$<1$ year & & $2.6(1.3$ to 5.2$)$ \\
\hline $1-5$ years & & 4.1 (1.8 to 9.2$)$ \\
\hline$>5$ years & & 5.5 (2.7 to 11.2$)$ \\
\hline Opioid dependence before OMT, years & 0.01 & \\
\hline 8 years (25th percentile) & & $3.3(2.0$ to 5.5$)$ \\
\hline 16 years ( 75 th percentile) & & 5.4 (3.1 to 9.4$)$ \\
\hline
\end{tabular}

Injecting-related treatment episodes were also substantially reduced (by $83 \%$ ) during OMT. This may have been due to those OMT patients who stopped injecting and to others who continued to inject but at a less frequent rate than before treatment. ${ }^{28}$ The reduction includes all kinds of injecting-related episodes among which local and systemic bacterial infections are by far the most frequent. The local skin infections often require surgical treatment and may give rise to severe systemic infections as septicaemia, fasciitis, endocarditis, osteomyelitis, discitis and others. Most of these conditions are rare, but they are much more frequent among injecting drug users than among the general population. ${ }^{36}$ Such infections may be life-threatening and may result in severe and permanent health problems, and often necessitate complex, long-lasting and expensive hospital treatment. When injecting-related health problems among drug users are considered, these apparently less serious conditions are often overshadowed by the focus on HIV and hepatitis C. However, some studies indicate that the burden on the healthcare system due to injecting-related local and systemic bacterial infections may be even greater than that due to bloodborne infections. ${ }^{37}$ The present study found that such bacterial infections were frequent among dependent opioid users and that they were substantially reduced during maintenance treatment.

Non-drug-related treatment episodes were found to increase (by 35\%) during OMT as compared to the pre-OMT period. Inpatient days increased by $98 \%$ and outpatient treatment contacts increased by $40 \%$. The reason for this may be closer contact with health services during maintenance treatment. Within the OMT programme, patients may receive healthcare that was not previously available to them, leading to the diagnosis and treatment of health problems that were previously not identified. ${ }^{38}$ If so, this increase in non-drug-related episodes may reflect improved access to health services and not an increase in morbidity. It is theoretically possible that this increase is due to adverse effects of OMT, but we have found no evidence of this in our scrutiny of the hospital records.

Drug-related treatment episodes and related inpatient days and outpatient hospital contacts were more frequent in the period after OMT compared to the periods before OMT and during OMT. These rates apply only to those patients who had left OMT. Although some patients may remain drug-free after leaving OMT, it is likely that many leave OMT, voluntarily or involuntarily, because of ongoing drug-taking, opposition to programme rules and control measures, or instability in taking their OMT medication. ${ }^{102531}$ Our data show that only $15 \%$ of those who left OMT were assessed as stable and drug-free at the time of leaving treatment. Patients with interrupted maintenance treatment may therefore constitute a patient subgroup with higher levels of risktaking behaviour and/or more serious health problems, and the post-OMT results may be influenced by selection bias. We therefore examined the interaction between OMT status (incidence rates before versus during OMT) and OMT cessation (having experienced OMT cessation or not) and found less reduction in drug-related treatment episodes in the cessation group compared to the group with continuous treatment Nevertheless, even the cessation group experienced a significant reduction in episodes of about $40 \%$. After OMT cessation, however, 
they experienced a more than fivefold increase compared to the period during OMT. The high postOMT incidence rates, therefore, reflect an increase in acute drug-related health problems after OMT cessation among patients with treatment interruption, and cannot be fully explained by selection effects. This is an important finding that should stimulate increased efforts to study the health effects of OMT cessation and to improve responses to minimise the harmful consequences.

Even the quartile taking most illegal drugs during OMT showed a significant $72 \%$ reduction in drug-related treatment episodes during versus before treatment. This indicates that not only patients with successful maintenance treatment, but also patients with poor rehabilitation results experience health improvement during OMT.

The present study focuses on acute/subacute hospital treatment episodes. Injecting drug users experience increased chronic morbidity that reduces their quality of life $^{83940}$ and some studies based on interviews ${ }^{41}$ and clinical assessment ${ }^{21}$ show a reduction in chronic health problems during OMT. However, considering the high mortality rate among injecting drug users,${ }^{10}$ the often life-threatening acute disease incidents comprise a substantial-and perhaps the most important-part of the morbidity pattern within this group.

The study has some limitations. First, the study assessed acute/subacute hospital-treated disease incidents and not morbidity as such. A key question is whether changes in incidence and treatment rates according to OMT status (before, during and after OMT) reflect a change in morbidity; in other words, whether these rates can be regarded as a proxy indicator for morbidity. Information on hospital treatment outside the local area was based on patient recall and some disease incidents prior to treatment may have been overlooked. In addition, closer contact with health services during OMT probably increases patients' helpseeking, resulting in hospital treatment for health problems that would not have been treated before OMT. Further, the patients were 5 years older during than before OMT, resulting in an age-dependent increase in morbidity. Even so, we found a significant reduction in treatment episodes during OMT. Therefore, our findings most probably reflect a 'true' reduction in acute/ subacute incidents of somatic disease, and hence in acute/subacute somatic morbidity, during compared to before OMT.

Another limitation is that primary healthcare contacts were not included in this study. In our previous study, ${ }^{24}$ however, general practitioner contacts were registered and we found that about $80 \%$ of all acute somatic disease incidents assessed as severe resulted in hospital treatment both before and during OMT. The focus on hospital treatment episodes should therefore provide an adequate assessment of OMT-related changes in severe acute health problems within the cohort. Also, the cohort is relatively old, with a mean age at first OMT entry of 37 years and mean duration of opioid dependence before the first admission to OMT of 12 years. This reflects the late introduction of OMT to Norway in 1998 with an accumulated demand for maintenance treatment by older patients at programme start and a high recommended age limit for OMT admittance during the first years. ${ }^{42}$ The cohort is typical of Norwegian OMT patients, but the age distribution is no doubt characterised by specific historical traits of the Norwegian OMT programme and this might be a possible limitation to the external validity of the study. However, we did check the effect modification of age at OMT entry (no significant interaction) and duration of opioid dependence before first OMT entry (less reduction among patients with fewer years of dependence) on the rates of drug-related treatment episodes before versus during OMT.

Yet another limitation is that the list of diagnoses to differentiate whether treatment episodes were drugrelated or not has not been validated by external researchers and we could find no similar list in the literature. There may be some uncertainty about whether some episodes were drug-related or not; however, inter-rater reliability scores were high. There were also analytical problems due to the fact that the post-OMT observation period was only 91 years versus 1000 before and 813 during OMT. This is mainly due to the high retention in OMT in Norway. The relatively few post-OMT years at risk is a limitation in the study, but with this design and the given retention in treatment, the post treatment period will nevertheless be unbalanced compared to the periods before and during OMT.

The study also has certain strengths. These include the high participation rate among patients in as well as out of maintenance treatment at the time of invitation, the access to all hospital records and the long observation period. The overall participation rate was high $(71 \%)$ and more than $99 \%$ of requested hospital records were obtained. Hence, selection bias was probably of limited importance in the study. Further, the evaluation of morbidity changes is based on in-depth assessment of full-text hospital records which could be expected to give more robust data than interview data or register data alone.

Despite possible limitations in the study design, the findings document a substantial reduction in acute and subacute drug-related disease incidents leading to hospital treatment during OMT compared to the period before OMT. This reduction in somatic morbidity during treatment seems also to be valid for patients with ongoing drug-taking during OMT. However, acute drugrelated health problems show an increase after OMT cessation, and this is a matter of concern. Further studies on somatic morbidity after OMT cessation should be carried out.

Author affiliations:

${ }^{1}$ Centre for Addiction Treatment, Oslo University Hospital, Oslo, Norway

${ }^{2}$ Norwegian Centre for Addiction Research, University of Oslo, Oslo, Norway 
${ }^{3}$ Department of General Practice, Institute of Health and Society, University of Oslo, Oslo, Norway

${ }^{4}$ National Addiction Centre, Department of Psychiatry, King's College London, London, UK

${ }^{5}$ The antibiotic centre for primary care, Institute of Health and Society, University of Oslo, Oslo, Norway

${ }^{6}$ Department of Internal Medicine, Innlandet Hospital, Gjoevik, Norway

${ }^{7}$ Department of Biostatistics, Institute of Basic Medical Sciences, University of Oslo, Oslo, Norway

Acknowledgements First we would like to thank all the participants for their kind cooperation. Further we would like to thank social workers, public health workers and pharmacy employees who recruited the participants. And lastly we would like to thank the hospitals, especially Innlandet Hospital and Oslo University Hospital, for giving access to record data. Axel Vetlesen and Lars Høiby (medical students at the University of Oslo) and Are Skeie Hermansen (sociology student at the University of Oslo) performed interviews (not authors).

Competing interests None.

Ethics approval This study was approved by the Regional Committee for Medical Research Ethics - Southern Norway (REK Sør) and the Norwegian Data Inspectorate.

Contributors IS had the original idea for the study, took part in the planning and developed the questionnaire and the list of diagnoses, carried out interviews, collected record data, performed data analyses and statistical analyses, took part in interpretation of data, drafted and is first author of the article, and is guarantor of the study. MB supervised the study and took part in the planning of the study, interpretation of data and revision of the article. MG contributed to the interpretation of data and took part in revision of the article. $M L$ supervised the study and took part in the planning of the study, interpretation of data and revision of the article. ER took part in the interpretation of record data and revision of the article. MT took part in the interpretation of data, planned and performed the statistical analyses and took part in the revision of the article. HW is project leader and the main supervisor of the study and participated in the planning of the study, interpretation of data and revision of the article.

Provenance and peer review Not commissioned; externally peer reviewed.

Data sharing statement Anonymised additional data are available from the corresponding author.

\section{REFERENCES}

1. Fischer B, Rehm J, Brissette S, et al. Illicit opioid use in Canada: comparing social, health, and drug use characteristics of untreated users in five cities (OPICAN study). $J$ Urban Health 2005;82:250-66.

2. Ross J, Teesson M, Darke S, et al. The characteristics of heroin users entering treatment: findings from the Australian treatment outcome study (ATOS). Drug Alcohol Rev 2005;24:411-18.

3. Popova S, Rehm J, Patra J, et al. Illegal drug-attributable morbidity in Canada 2002. Drug Alcohol Rev 2007;26:251-63.

4. Bijl RV, Ravelli A, van Zessen G. Prevalence of psychiatric disorder in the general population: results of The Netherlands Mental Health Survey and Incidence Study (NEMESIS). Soc Psychiatry Psychiatr Epidemiol 1998;33:587-95.

5. Kessler RC, McGonagle KA, Zhao S, et al. Lifetime and 12-month prevalence of DSM-III-R psychiatric disorders in the United States. Results from the National Comorbidity Survey. Arc Gen Psychiatry 1994:51:8-19.

6. Regier DA, Farmer ME, Rae DS, et al. Comorbidity of mental disorders with alcohol and other drug abuse. Results from the Epidemiologic Catchment Area (ECA) Study. JAMA 1990;264:2511-18.

7. Millson PE, Challacombe L, Villeneuve PJ, et al. Self-perceived health among Canadian opiate users: a comparison to the general population and to other chronic disease populations. Can J Public Health 2004;95:99-103.

8. Puigdollers E, Domingo-Salvany A, Brugal MT, et al. Characteristics of heroin addicts entering methadone maintenance treatment: quality of life and gender. Subst Use Misuse 2004;39:1353-68.

9. Brugal MT, Domingo-Salvany A, Puig R, et al. Evaluating the impact of methadone maintenance programmes on mortality due to overdose and aids in a cohort of heroin users in Spain. Addiction 2005;100:981-9.
10. Darke S, Degenhardt L, Mattick R. Mortality Amongst Illicit Drug Users: Epidemiology, Causes and Intervention. Cambridge: Cambridge University Press, 2007.

11. Reimer J, Lorenzen J, Baetz B, et al. Multiple viral hepatitis in injection drug users and associated risk factors. $J$ Gastroenterol Hepatol 2007;22:80-5.

12. Taylor A, Goldberg D, Hutchinson S, et al. Prevalence of hepatitis $C$ virus infection among injecting drug users in Glasgow 1990-1996: are current harm reduction strategies working? J Infect 2000;40:176-83.

13. Hope V, Kimber J, Vickerman P, et al. Frequency, factors and costs associated with injection site infections: findings from a national multi-site survey of injecting drug users in England. BMC Infect Dis 2008;8:120.

14. Bargagli AM, Hickman M, Davoli M, et al. Drug-related mortality and its impact on adult mortality in eight European countries. Eur $J$ Public Health 2006;16:198-202.

15. WHO. Guidelines for the Psychosocially Assisted Pharmacological Treatment of Opioid Dependence. Geneva: World Health Organization, 2009.

16. Fischer B, Rehm J, Kim G, et al. Eyes wide shut?-A conceptual and empirical critique of methadone maintenance treatment. Eur Addict Res 2005;11:1-9; discussion 10-14.

17. Darke S, Ross J, Teesson M. The Australian Treatment Outcome Study (ATOS): what have we learnt about treatment for heroin dependence? Drug Alcohol Rev 2007;26:49-54.

18. Gowing L, Farrell M, Bornemann R, et al. Substitution treatment of injecting opioid users for prevention of HIV infection. Cochrane Database Syst Rev 2008;(2):CD004145.

19. Dole VP, Nyswander ME, Kreek MJ. Narcotic blockade-a medical technique for stopping heroin use by addicts. Trans Assoc Am Physicians 1966;79:122-36.

20. Kreek MJ. Tolerance and dependence: implications for the pharmacological treatment of addiction. In: Harris LS, ed, NIDA Research Monograph 76-Problems of Drug Dependence 1986: Proceeding of the 48th Annual Scientific Meeting The Committee On Problems of Drug Dedpendence. Rockville: US Department of Health and Human Services, 1987:53-62.

21. Wittchen HU, Apelt SM, Soyka M, et al. Feasibility and outcome of substitution treatment of heroin-dependent patients in specialized substitution centers and primary care facilities in Germany: a naturalistic study in 2694 patients. Drug Alcohol Depend 2008;95:245-57.

22. Stenbacka M, Leifman A, Romelsjo A. The impact of methadone on consumption of inpatient care and mortality, with special reference to HIV status. Subst Use Misuse 1998;33:2819-34.

23. Romelsjo A, Engdahl B, Stenbacka M, et al. Were the changes to Sweden's maintenance treatment policy 2000-06 related to changes in opiate-related mortality and morbidity? Addiction 2010;105:1625-32.

24. Skeie I, Brekke M, Lindbaek M, et al. Somatic health among heroin addicts before and during opioid maintenance treatment: a retrospective cohort study. BMC public health 2008:8:43

25. Waal H, Clausen T, Håseth A, et al. Seraf Rapport 1/2010 (revidert utgave) Siste år med gamle retningslinjer Statusrapport 2009. Oslo: Norwegian Centre for Addiction Research, 2010.

26. Clausen T, Anchersen K, Waal H. Mortality prior to, during and after opioid maintenance treatment (OMT): a national prospective crossregistry study. Drug Alcohol Depend 2008;94:151-7.

27. Fugelstad A, Stenbacka M, Leifman A, et al. Methadone maintenance treatment: the balance between life-saving treatment and fatal poisonings. Addiction 2007;102:406-12.

28. Kimber J, Copeland L, Hickman M, et al. Survival and cessation in injecting drug users: prospective observational study of outcomes and effect of opiate substitution treatment. BMJ 2010;341: c3172.

29. Caplehorn JR, Dalton MS, Haldar F, et al. Methadone maintenance and addicts' risk of fatal heroin overdose. Subst Use Misuse 1996;31:177-96.

30. Dolan KA, Shearer J, White B, et al. Four-year follow-up of imprisoned male heroin users and methadone treatment: mortality, re-incarceration and hepatitis $\mathrm{C}$ infection. Addiction 2005; $100: 820-8$.

31. Gronbladh L, Ohlund LS, Gunne LM. Mortality in heroin addiction: impact of methadone treatment. Acta Psychiatr Scand 1990;82:223-7.

32. Zanis DA, Woody GE. One-year mortality rates following methadone treatment discharge. Drug Alcohol Depend 1998;52:257-60.

33. Cornish R, Macleod J, Strang J, et al. Risk of death during and after opiate substitution treatment in primary care: prospective observational study in UK General Practice Research Database. BMJ 2010;341:c5475. 
34. Davoli M, Bargagli AM, Perucci CA, et al. Risk of fatal overdose during and after specialist drug treatment: the VEdeTTE study, a national multi-site prospective cohort study. Addiction 2007:102:1954-9.

35. Degenhardt L, Randall D, Hall W, et al. Mortality among clients of a state-wide opioid pharmacotherapy program over 20 years: risk factors and lives saved. Drug Alcohol Depend 2009;105:9-15.

36. Spijkerman IJ, van Ameijden EJ, Mientjes GH, et al. Human immunodeficiency virus infection and other risk factors for skin abscesses and endocarditis among injection drug users. J Clin Epidemiol 1996;49:1149-54.

37. Hope VD, Marongiu A, Parry JV, et al. The extent of injection site infection in injecting drug users: findings from a national surveillance study. Epidemiol Infect 2010;138:1510-18.

38. Godfrey C, Stewart D, Gossop M. Economic analysis of costs and consequences of the treatment of drug misuse: 2-year outcome data from the National Treatment Outcome Research Study (NTORS). Addiction 2004;99:697-707.

39. Millson P, Challacombe L, Villeneuve PJ, et al. Determinants of health-related quality of life of opiate users at entry to low-threshold methadone programs. Euro Addict Res 2006;12:74-82.

40. Cullen W, O'Brien S, O'Carroll A, et al. Chronic illness and multimorbidity among problem drug users: a comparative cross sectional pilot study in primary care. BMC Fam Pract 2009;10:25.

41. Williamson A, Darke S, Ross J, et al. Changes and predictors of change in the physical health status of heroin users over 24 months. Addiction 2009;104:465-70.

42. Waal H. Merits and problems in high-threshold methadone maintenance treatment. Evaluation of medication-assisted rehabilitation in Norway 1998-2004. Euro Addict Res 2007;13:66-73. 